Editorial

\title{
Era de la Cuarta Revolución Industrial
}

\section{Age of the Fourth Industrial Revolution}

\author{
Gloria Piedad Gasca-Hurtado ${ }^{1}$, Liliana Machuca-Villegas ${ }^{1,2}$ \\ gpgasca@udem.edu.co, liliana.machuca@correounivalle.edu.co \\ ${ }^{1}$ Universidad de Medellín, Carrera 87 No. 30-65, 50026, Medellín, Colombia \\ ${ }^{2}$ Universidad del Valle, Calle 13 \# 100-oo, 760032, Cali, Valle del Cauca, Colombia
}

DOI: 10.17013/risti.34.o

\section{Introducción}

Los artículos presentados en este número fueron seleccionados de los trabajos aceptados en la Décimo Segunda Edición del Seminario Internacional de Ciencias de la Computación (SICC) 2019, realizado en la Ciudad de Medellín, Antioquia, Colombia durante los días 23, 24 y 25 de octubre del 2019. Este seminario ha tenído una tasa de acpetación del 16.32\% de los artículos enviados.

En este número de la Revista Ibérica de Sistemas y Tecnologías de la Información (RISTI), se abordan temas relacionados con la Era de la Cuarta Revolución Industrial. Los trabajos de este número son las versiones originales presentadas por los autores en comunicación oral durante el congreso SICC 2019 y que fueron seleccioandos después de realizarse un proceso evaluación por pares doble ciego.

\section{Cuarta revolución industrial}

El término Industria 4.0 o Cuarta Revolución Industrial se refiere a una amplia gama de conceptos. Clasificar dichos conceptos y relacionarlos a una sola disciplina, así como distiguirlos específicamente es difícil (Lasi, Fettke, Kemper, Feld, \& Hoffmann, 2014). Sin embargo, es posible tener en cuenta algunos conceptos fundamentales que se destacan al referirse a la Cuarta Revolución Industrial. Estos conceptos fundamentales se orientan en la definición de la industria 4.o en el marco de Fábricas Inteligentes o Smart Factory, Tecnología Inteligente o Smart Inteligent, pasando por sistemas ciberfísicos, que hacen referencia a la fusión del nivel físico y el digital a tal nivel que la representación física y 
digital no puede diferenciarse de manera razonable. También se consideran temas de responsabilidad social a nivel corporativo y ético, con el fin de tener en cuenta aspectos relacionados con el impacto negativo social, ambiental o económico que puede llegar a afectar a las personas y al planeta (Sanchez, 2019).

En este contexto es posible afirmar que los enfoques e ideas en el ámbito de la Cuarta Revolución Industrial están situados entre disciplinas como ingeniería eléctrica, administración de empresas, informática, tecnología y software, negocios e información, ingeniería de software e ingeniería mecánica.

En términos generales, con el surgimiento de la Cuarta Revolución Industrial como un requisito de la red de producción global, las empresas y los países están conectados más estrechamente a través de redes de cadena de suministro en todo el mundo (Lasi et al., 2014).

La Cuarta Revolución Industrial significa que todas las innovaciones en tecnología digital maduran, especialmente en el sector manufacturero, durante el proceso de transformación. Elementos relacionados con tecnologías como Internet especialmente avanzada, inteligencia artificial y robótica, tecnología avanzada de sensores, tecnología en la nube que acelera la transferencia y utilización de datos, fabricación digital (impresión 3D), nuevos servicios y modelos de comercialización, teléfonos inteligentes y algoritmos para herramientas de navegación, entrega y transporte, servicios y vehiculos autonomos, están incluidos en la cadena de valor global para que puedan ser compartidos por muchas compañías de muchos países (Cakmakci, 2019).

Por lo tanto, en la Cuarta Revolución Industrial, hay diferentes tecnologías que se unen de distintas maneras pero con el mismo fin. En este sentido, no solo cambia el mundo a nivel industrial sino que las mismas organizaciones deben cambiar. Gestionar las organizaciones globales para que enfrenten los retos de ser globales en esta nueva Era de la Cuarta Revolución Industrial es otro de los cambios imparables. Las organizaciones deben desarrollar mejores capacidades para ser interconectadas e internacionales. Deben lograr definir formas de revolucionarse con el mundo e impactar, cambiando incluso el enfoque de gestión de proyectos para afrontar retos relacionados con la Industria 4.0 (Cakmakci, 2019).

Como resultado, se espera un cambio industrial que va desde la orientación del producto o servicio que se genera hasta su relación con el mundo que la rodea, incluso en las industrias más tradicionales. Es posible visualizar en un futuro cercano la aparición de nuevos tipos de empresas que adopten nuevos roles específicos dentro del proceso de fabricación.

Con la planificación, análisis, modelado, diseño, implementación y mantenimiento (en resumen: el desarrollo) de sistemas de información tan complejos, dinámicos e integrados, surge una tarea atractiva y al mismo tiempo desafiante para las disciplinas que se incluyen hoy por hoy en esta revolución industrial, que puede asegurar y desarrollar aún más la competitividad de las empresas industriales.

La Industria 4.0 se centra en el establecimiento de productos inteligentes y procesos de producción. Por lo tanto, es necesario buscar alternativas para hacer frente a la necesidad de un rápido desarrollo de productos, una producción flexible y entornos 
complejos (Vyatkin, Salcic, Roop, \& Fitzgerald, 2007)(Brettel, Friederichsen, Keller, \& Rosenberg, 2014). Por consiguiente, se habla de entornos de fabricación inteligente. Los productos inteligentes y personalizados comprenden el conocimiento de su proceso de fabricación y la aplicación del consumidor, además, lideran de forma independiente su camino a través de la cadena de suministro (Henning, 2013) (Brettel et al., 2014).

En resumen, los temas de la Cuarta Revolución Industrial son de gran importancia para la academia, a tal punto que el interés de la ciencia y la investigación en el área de las Ciencias de la Computación no es ajena al aporte que se debe realizar. Por lo tanto, hacen parte de los avances investigativos que se presentan en los trabajos seleccionados para ser publicados en este número de RISTI. Estos trabajos han sido seleccionados porque abordan temáticas de interés en la actualidad y se enmarcan en soluciones del mundo real con un enfoque asociado a la Era de la Cuerta Revolución Industrial.

\section{Estructura}

En el primer artículo los autores presentan una propouesta enfocada a la educación y hacen énfasis en los resultados obtenidos a partir del diseño, implementación y validación de una herramienta de software $3 \mathrm{D}$, que permitió configurar robots Lego Mindstorms EV3 y VEX Robotics EDR, para cumplir una tarea específica. Con esta propuesta se pretende propiciar la adquisición de competencias técnicas tan necesarias en la Era de la Cuerta Revolución Industrial alrededor de temas como electrónica, informática, mecánica, y otras competencias transversales como el trabajo en equipo, la resolución de problemas y el pensamiento crítico. De los aspectos más relevantes de esta investigación está el uso de robots educativos, teniendo en cuenta de manera esencial el contexto y el usuario final del robot para lograr una solución. Específicamente esta propuesta tiene en cuenta los requerimientos iniciales del usuario del robot para, mediante el uso de la robótica pedagógica, solucionar una necesidad. Esta alternativa se convierte en el punto de partida de una educación que verdaderamente logre un impacto y un cambio de mentalidad, aprovechando los escenarios y la infraestructura existentes.

En el segundo artículo se presenta un enfoque de descripción de imágenes desde la perspectiva semántica y se evalúa mediante un análisis léxico-sintáctico para comprobar que las descripciones dadas sean parte del lenguaje definido. Dentro de los resultados obtenidos, los más relevantes muestran que, al tomar en cuenta las imágenes como un todo y no como objetos aislados se puede obtener información importante para describirla, incluso podrían realizarse inferencias sobre lo que sucede en la escena que conforma la imagen, por ello es muy importante la verificación sintáctica ya que de esta manera se evita añadir ruido al sistema y se trabaja solo con elementos válidos. Además, se muestra la validación sintáctica la cual realiza una verificación de las clases para solo procesar las válidas y de esta manera evitar añadir ruido al sistema con palabras que no son clases reconocidas tales como: conectores, numerales, etc.

En el tercer artículo se plantea un proceso de descubrimiento de conocimiento en el texto no estructurado de las historias clínicas del área de traumatología del Hospital San Vicente Fundación mediante minería de texto. Para el estudio se aplicaron técnicas de preparación de texto como eliminación de palabras no relevantes, sustitución de términos, eliminación de acentos y derivación de palabras. El resultado obtenido es la 
conformación de un modelo de las palabras más relevantes en los registros clínicos del Hospital en el área de traumatología.

En el cuarto artículo los autores buscan mejorar la toma de decisiones de revisiones de artículos científicos a partir de la evaluación del revisor, es decir, predecir si un artículo científico debería ser aceptado o rechazado en función de los comentarios aportados por los revisores. Además, con su propuesta, los autores buscan lograr cuantificar la consistencia entre los comentarios de un revisor con respecto a su evaluación, usualmente expresada en una escala multiclase. Aunque con varios aspectos por mejorar, el trabajo muestra resultados alentadores, dado que presenta un beneficio cuantitativo de un aumento significativo en el tamaño de los datos y a la vez un mejor rendimiento de un clasificador basado en los datos aumentados en comparación a uno basado solo en los datos originales.

El quinto artículo aborda el problema de la transmisión de datos en redes de sensores inalámbricas (WSN), con garantías de tiempo real. Este es un tema que tiene referentes importantes en la comunidad científica internacional, pero que aún no dispone de una solución, que satisfaga este requerimiento completamente. El trabajo que se presenta en este artículo está relacionado con un método (algoritmo) de planificación para la transmisión de datos en tiempo real con redes de sensores inalámbricas (WSN), basado en técnicas de planificación estática y aplicado a redes que utilicen espacios de tiempo (time-slot) para la transmisión de datos en eventos periódicos, donde se garantice un tiempo acotado de transmisión.

El sexto artículo tiene como propósito la implementación de un controlador difuso en línea, empleando una plataforma del internet de las cosas (IoT), para obtener un conjunto de datos que se procesan, empleando redes neuronales, con el objetivo de obtener un modelo que permita la identificación de la dinámica de un sistema térmico. Con este trabajo se pretende evidenciar que la aplicación de herramientas de la inteligencia artificial y el internet de las cosas en contextos industriales traerá beneficios en el desarrollo de procesos con sistemas más eficientes y con capacidad de autogestión. Lo anterior considerando que la Cuarta Revolución Industrial trae consigo nuevas oportunidades en creación de productos y servicios dando paso a trabajos que requieren nuevos conocimientos, además de un alto componente de creatividad e innovación. Sobre todo, cuando el mundo en general busca ser cada vez más competitivo y tener una industria que satisfaga los requerimientos modernos.

El séptimo artículo está relacionado con la competividad que las empresas de desarrollo de software buscan, respondiendo justamente a la exigencia de la Cuarta Revolución Industrial a nivel de satisfacción de requisitos. Por lo anterior, hoy en día, organizaciones como la ISO están enfocadas en el desarrollo de estándares que impulsen su competitividad, reforzando el uso de prácticas probadas de ingeniería de software y sean adecuados a las características específicas de este tipo de empresas, como lo es la serie de estándares ISO/IEC 29110. El objetivo de los autores es realizar una comparativa entre el estado del arte y el estado de la práctica referente a la implementación de dicho estándar, en entidades muy pequeñas. Los resultados más importantes muestran, que las principales debilidades están relacionadas con la falta de ejecución de actividades de verificación y validación, y el desarrollo y/o documentación de casos y procedimientos de prueba. 
Finalmente, el octavo artículo está muy relacionado con la búsqueda de lograr calidad y competividad en esta Era de la Cuarta Revolución Industrial. Este trabajo estudia mecanismos de medición de la calidad del diseño arquitectural de software ya que se ha identificado una carencia en métricas de calidad, sobre todo en aquellas enfocadas en medir el factor de flexibilidad y de herencia de implementación de una arquitectura de clases. Por lo tanto, los autores de este trabajo proponen un conjunto de cinco métricas de calidad, tres de ellas son para medir el factor de herencia de implementación, son: Factor de Herencia de Implementación (FHI), Factor de Herencia de Implementación por Jerarquía de Clases (FHIJ) y Factor Herencia de Implementación de una Arquitectura de Clases (FHIAC); las dos restantes son para medir el factor de flexibilidad, estas son: Factor de Flexibilidad de Clase (FFC) y Factor Medio de Flexibilidad de Clases(FMFC).

\section{Agradecimientos}

Agradecemos al director de la Revista Álvaro Rocha por otorgar la oportunidad para dar a conocer los trabajos más relevantes presentados en el seminario SICC 2019. Asimismo, expresamos nuestra gratitud a los autores de los trabajos publicados, lo cual hizo posible este número en RISTI. Aunando esfuerzos queremos agradecer a las empresas y universidades aliadas que con su aporte lograron engrandecer la Décimo Segunda Edición del Seminario Internacional de Ciencias de la Computación.

\section{Referencias}

Brettel, M., Friederichsen, N., Keller, M., \& Rosenberg, M. (2014). How virtualization, decentralization and network building change the manufacturing landscape: An Industry 4.0 Perspective. International Journal of Mechanical Industrial Science and Engineering, 8(1), 37-44.

Cakmakci, M. (2019). Interaction in Project Management Approach Within Industry 4.o. In Advances in Manufacturing II (pp. 176-189). Springer.

Henning, K. (2013). Recommendations for implementing the strategic initiative INDUSTRIE 4.O. Germany: Federal Ministry of Education and Research.

Lasi, H., Fettke, P., Kemper, H.-G., Feld, T., \& Hoffmann, M. (2014). Industry 4.o. Business \& Information Systems Engineering, 6(4), 239-242.

Sanchez, D. O. M. (2019). Sustainable Development Challenges and Risks of Industry 4.0: A literature review. In 2019 Global IoT Summit (GIoTS) (pp. 1-6).

Vyatkin, V., Salcic, Z., Roop, P. S., \& Fitzgerald, J. (2007). Now that's smart! Industrial Electronics Magazine. IEEE, 1(4), 17-29. 